\title{
Neuroimaging of emotion: empirical effects of proportional global signal scaling in fMRI data analysis
}

\author{
Markus Junghöfer, ${ }^{\mathrm{a}, \mathrm{b}, *}$ Harald T. Schupp, ${ }^{\mathrm{a}}$ Rudolf Stark, ${ }^{\mathrm{c}}$ and Dieter Vaitl ${ }^{\mathrm{c}}$ \\ ${ }^{a}$ Department of Psychology, University of Konstanz, Germany \\ ${ }^{\mathrm{b}}$ Institute for Biomagnetism and Biosignalanalysis, Münster University Hospital, Germany \\ ${ }^{\mathrm{c}}$ Department of Psychology, University of Giessen, Germany
}

Received 2 June 2004; revised 19 November 2004; accepted 7 December 2004

Available online 2 February 2005

\begin{abstract}
Global variations of BOLD-fMRI signal are often considered as nuisance effects. This unwanted source of variance is commonly eliminated using proportional global signal scaling (PGSS). However, application of PGSS relies on the assumption that global variations of BOLD signal and experimental conditions are uncorrelated. It has been shown for cognitive tasks that the unjustified application of PGSS might greatly distort statistical results. The present study examined this issue in the domain of emotion research. Specifically, fMRI data were obtained in a block-design, while 21 subjects passively viewed high and low emotionally arousing pleasant, unpleasant, and neutral pictures. Violations of the orthogonality assumption were found for analyses of emotional pictures high in arousal, causing dramatically different outcomes when compared to analyses performed without PGSS. Application of PGSS was associated with attenuated emotional activation in visual cortical areas, insensitivity to emotional activations in limbic and paralimbic regions, and widely distributed artificial deactivations. In contrast, the orthogonality assumption was not violated for low arousing emotional materials. Thus, the validity of using PGSS varied as a function of the emotional arousal of the stimuli. Taken together, the unwarranted use of PGSS might contribute to conflicting results in affective neuroscience fMRI studies, in particular with respect to limbic and paralimbic structures.

(C) 2004 Elsevier Inc. All rights reserved.
\end{abstract}

Keywords: fMRI; Global signal scaling; Emotion; Affect

\section{Introduction}

Blood oxygen level dependent (BOLD) functional magnetic resonance imaging (fMRI) is increasingly used to examine the functional neuroanatomy of emotion (e.g., Davis and Whalen, 2001; Pessoa et al., 2002; Phan et al., 2002). However, methodological issues already critically discussed in cognitive fMRI research might be of particular concern when studying emotional

* Corresponding author. Department of Psychology, University of Konstanz, PO Box D36, 78457 Konstanz, Germany. Fax: +49 7531 882971.

E-mail address: Markus.Junghöfer@uni-konstanz.de (M. Junghöfer).

Available online on ScienceDirect (www.sciencedirect.com). processes. Specifically, one unresolved controversy is the use of proportional scaling of global BOLD signals (PGSS) in fMRI analysis. The present report tested the underlying validity of using PGSS in fMRI studies of emotional reactivity, and determined the potential consequences introduced by its unjustified use.

Global variations of BOLD-fMRI signal are defined as changes common to the entire brain volume, and have been considered to reflect background activity rather than signal changes related to experimental manipulations (Ramsay et al., 1993). Consequently, global variations of BOLD-fMRI signal are commonly considered nuisance effects, contributing unwanted sources of variance such as hardware scanner drifts, physiological noise or pulsations. While most accept that there is a fundamental necessity for global signal correction, discussion centers on the proper method of normalization, and the identification of global signal changes as either 'additive' or 'multiplicative' compared to regional effects of interest (Arndt et al., 1996; Fox et al., 1988; Friston et al., 1990). However, normalization of global signal changes might result in a confound, i.e., significantly altering the outcome of an analysis when global signal is not orthogonal to the experimental paradigm (Aguirre et al., 1998). Observing a strong positive correlation of a speeded reaction time task and global BOLD signal, this concern was empirically bolstered by Aguirre et al. (1998). As a consequence of the unjustified application of PGSS, the statistical findings of the BOLD signal analysis was greatly distorted: Effects of interest were attenuated in the PGSS analysis compared to the analysis without global scaling, and in some cases the PGSS-based analysis revealed significant deactivations in structures of interest. Desjardins et al. (2001) made similar observations using an oddball task and a working memory manipulation. Taken together, the application of global signal correction in fMRI analysis has been questioned in cognitive research on similar grounds as in analyses of positron emission tomography (PET; cf. Andersson, 1997; Strother et al., 1995).

Reservations regarding the use of PGSS were explored here in the domain of emotion research. Exploring this issue in the visual modality is particularly well suited to this task, given that many fMRI studies have examined the processing of visually presented emotional stimuli (cf. Davis and Whalen, 2001; Phan et al., 2002). 
Previous studies consistently observe enhanced activation in the visual cortex for emotional compared to neutral pictures taken from the International Affective Picture System (IAPS, e.g., Lang et al., 1998, 1999; Sabatinelli et al., 2004) as well as pictures of emotional and neutral facial expressions (e.g., Pessoa et al., 2002; Vuilleumier et al., 2001; Whalen et al., 2001). Activations were observed in several areas implicated in visual information processing, including the gyrus occipitalis, gyrus fusiformis, and superior temporal sulcus, when emotional and neutral contents were contrasted. Furthermore, a recent study observed that the signal changes in the visual cortex varied strongly with emotional arousal. Specifically, pleasant and unpleasant pictures high in arousal were associated with strong and widely distributed visual cortical activations compared to low arousing emotional or neutral materials (Bradley et al., 2003). As a consequence, the strong and distributed activations elicited by highly arousing emotional contents might be expected to dominate the global signal variance. Thus, the orthogonality assumption of global signal and experimental condition might be violated when contrasting visual processing of highly arousing emotional with neutral stimuli.

The present study examined the orthogonality assumption underlying the use of PGSS by contrasting BOLD signal activations evoked by visual processing of pictures varying in emotional arousal, and, if violated, determined the consequences associated with its unjustified application. If emotional picture processing is correlated with global signal variance, we expected to see similar detrimental effects of PGSS application as has been observed in cognitive tasks (cf. Aguirre et al., 1998; Desjardins et al., 2001). In addition, considering the modest effect sizes of emotional activations in limbic and paralimbic structures (cf. Davis and Whalen, 2001; Phan et al., 2002), these areas might be particularly susceptible to the unjustified application of PGSS. Finally, hemispheric and gender differences have been of particular interest in emotion research (e.g., Hamann et al., 2004; Sabatinelli et al., 2004; Wager et al., 2003). We therefore included these factors in our analyses with and without PGSS.

\section{Methods}

Twenty-one subjects (10 males) viewed a series of pictures taken from the International Affective Picture System (IAPS; Lang et al., 1999). Based on normative ratings of emotional valence and arousal, 500 pictures were divided equally into five picture categories-high arousal pleasant, low arousal pleasant, neutral, low arousal unpleasant, and high arousal unpleasant. Picture parameters such as brightness, contrast, color distribution, physical complexity or spatial frequencies did not significantly differ across the picture categories. Pictures were presented in 2 sessions, comprising 100 blocks of $12 \mathrm{~s}$ each, with individual picture durations of either $333 \mathrm{~ms}$ or $1333 \mathrm{~ms}$, without perceivable interpicture intervals. Picture blocks were presented pseudo-randomly, with the restriction that blocks of identical categories could not follow each other. Picture block order was balanced across subjects. Subjects were asked to passively view the stimuli while keeping their eyes focused on a central fixation cross.

Functional images were acquired on a 1.5-T Siemens Magnetom Symphony scanner equipped with Quantum gradients. A standard T1-weighted high-resolution whole brain sagittal anatomical scan (in plane matrix: $256 \times 256$; voxel size $0.7 \times 0.7 \times 6.5 \mathrm{~mm}$ ) was obtained at the beginning of the session. Afterwards, T2*-weighted, gradient echo, echo planar images (EPI) were acquired for 2 sessions of 20 min each $(20$ blocks per category $\times 5$ categories $\times 12 \mathrm{~s}$; TR $=$ $\left.3000 \mathrm{~ms}, \mathrm{TE}=60 \mathrm{~ms}, \alpha=90^{\circ}\right)$. Each functional scan consisted of 20 transverse slices (in plane matrix: $64 \times 64 ; 3 \times 3 \mathrm{~mm}$ ) with a thickness of $5 \mathrm{~mm}$-no gap-oriented parallel to the AC-PC line.

The functional images were analyzed using Statistical Parametric Mapping (Friston et al., 1995; SPM99). The first four scans were discarded to allow for signal stabilization, and the remaining volumes were slice-time corrected and realigned. Images were then spatially normalized to the Montreal Neurological Institute (MNI) standard brain, and resampled to a $2-\mathrm{mm}$ isotropic voxel size. All volumes were smoothed with an $8 \times 8 \times 10 \mathrm{~mm}$ full width half maximum Gaussian kernel. The high-pass cutoff period were set $192 \mathrm{~s}(\sim 0.005 \mathrm{~Hz})$, and a Gaussian filter with $4 \mathrm{~s}$ cutoff period was chosen as low-pass filter $(0.25 \mathrm{~Hz})$. The experimental conditions were modeled using a boxcar function convolved with an approximated hemodynamic response function.

In order to test for independency of global signal and specified contrasts of interest, correlations of global BOLD signal and an approximated hemodynamic response function were calculated for each picture block in each subject. Subsequently, the resulting correlation coefficients were entered as dependent variables in analyses of variance for dependent measures (Rosenthal and Rosnow, 1985; Scheffé, 1959) and tested for the following three predefined contrasts of interest: "High Arousing Emotional vs. Neutral"; "Low Arousing Emotional vs. Neutral"; and "High Arousing Pleasant vs. Neutral".

Thus, the orthogonality assumption has been tested with regard to selected contrasts of interest rather than individual conditions. Since experimental conditions may evince a positive or negative correlation with the global signal, it is conceivable that global signal covariations for selected contrasts may exceed the correlation of global signal for individual conditions. In addition, tests for orthogonality were based on preprocessed data rather than raw data. Preprocessing of the fMRI signal might reduce noise in the data and thereby increase correlations of global signal and selected contrasts (or conditions). Thus, the orthogonality assumption was tested here with regard to selected contrasts of interest based on the preprocessed data entering the main analyses.

Effects of the application of PGSS were assessed separately for low and high emotional arousal pictures. Specifically, a first set of analyses considered highly arousing pleasant, neutral, and highly arousing unpleasant pictures, modeling the condition effects according to the general linear model and a linear trend of increasing ('activation') or decreasing ('deactivation') BOLD signal changes with increasing emotional arousal $(+1,-2,+1){ }^{1}$ A second set repeated these analyses, but included only low arousing emotional and neutral pictures. Both sets of analysis were

\footnotetext{
${ }^{1}$ The terms 'activation' and 'deactivation' are used for brevity: The given contrasts do not distinguish between increasing BOLD with decreasing emotional arousal and decreasing BOLD with increasing arousal. Areas of 'deactivations' are, therefore, either less activated by emotional pictures or were strongly activated by neutral stimuli. Furthermore, Desjardins et al. (2001) suggested global signal orthogonalization with respect to the non-constant covariates of the experimental design matrix. PGSS based on such an 'adjusted global signal' would only correct for the part of the global signal, which is not task correlated. However, as differences between the results of 'adjusted' PGSS and the analysis without PGSS were small with regard to the dramatic effects comparing both with traditional PGSS, results of the 'adjusted' PGSS analysis have been omitted for brevity.
} 
performed on the two streams of statistical analysis with and without PGSS. Furthermore, in order to provide a more direct comparison of the effects of global signal scaling, second-level, two-sample $t$ tests comparing the contrast analysis results of all subjects with and without PGSS were performed for the two sets of contrasts (highly arousing emotional vs. neutral and low arousing emotional vs. neutral).

Further exploratory analysis served to determine the impact of the unjustified application of PGSS on hemispheric and gender differences. For instance, Sabatinelli et al. (2004) observed an increased activation in visual processing areas in men compared to women, contrasting high-arousing pleasant and neutral scenes. Therefore, to exemplify PGSS effects on gender differences, twosample $t$ tests contrasting the group of 10 males with the sample of 11 females were calculated for the contrast of highly arousing pleasant vs. neutral contents. Similarly, previous findings consistently suggest the increased engagement of right-hemispheric visual processing areas for highly emotional materials (cf. Bradley et al., 2003). Therefore, the impact of PGSS on hemispheric asymmetries was explored using paired $t$ tests contrasting the highly arousing emotional vs. neutral contrast effects with their corresponding laterally transposed images.
Results

High arousing emotional vs. neutral picture contrasts

Highly arousing picture perception led to significantly stronger global BOLD signal than neutral picture perception $[F(1,42)=$ 21.6; $P<0.00001]$.

In line with previous reports (cf. Aguirre et al., 1998), this violation of the orthogonality assumption resulted in widely differing outcomes comparing the two streams of fMRI analysis with and without PGSS: As shown in Fig. 1A and Table 1, the application of global signal scaling led to an attenuated effect of emotional activation in structures with a positive correlation of local and global BOLD signal ("activation"; indicated by red-hued colors). Omitting global signal scaling, structures associated with pronounced BOLD signal activations during highly arousing versus neutral picture conditions were apparent in uni- and heteromodal processing areas in the occipital, parietal, and temporal lobes. Invariably, although still significant, application of PGSS was reflected by reduced effect sizes and cluster volumes. Perhaps more importantly, both analyses also differed qualitatively, i.e., revealing significant findings in analyses without PGSS when
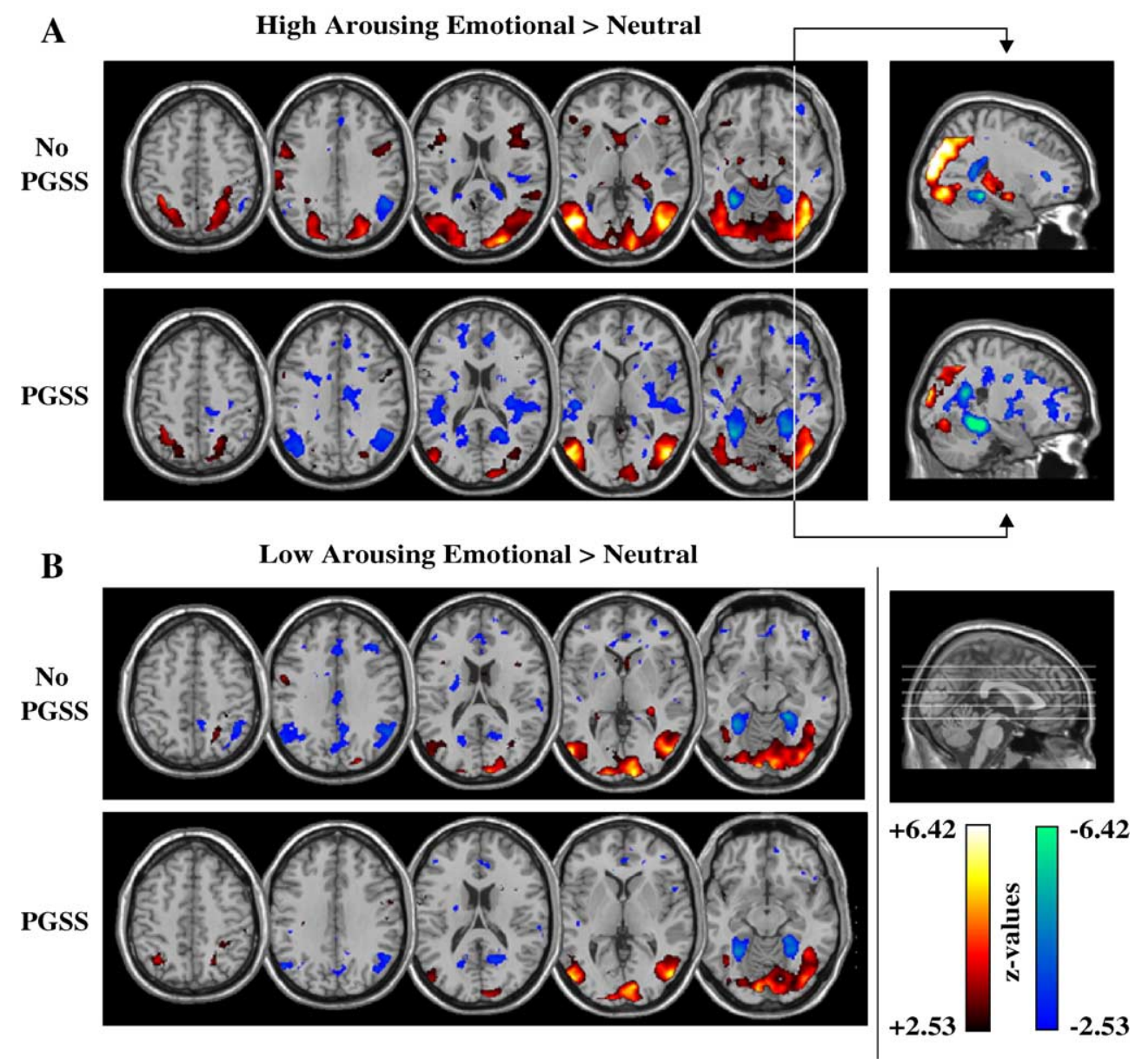

Fig. 1. Random effect analysis across 21 subjects $(k=20$; DF $=20)$. Significant BOLD signal differences contrasting high arousing emotional (A) or low arousing emotional (B) with neutral picture conditions based on an analysis without (top columns) or with PGSS (bottom columns). Red-hued colors indicate a stronger BOLD signal for the emotional compared to the neutral stimuli; blue-hued colors indicate stronger activations for neutral compared to emotional picture conditions. 
Table 1

Cluster sizes of correlated BOLD activation (left) or deactivation (center) contrasting "High Arousing Emotional vs. Neutral" picture conditions (as illustrated in Fig. 1A) for both streams of fMRI analysis with and without global signal covariates

\begin{tabular}{|c|c|c|c|c|c|c|c|c|c|c|c|c|c|}
\hline & \multirow[t]{3}{*}{ Structure } & \multicolumn{4}{|c|}{ "Activation" } & \multicolumn{4}{|c|}{ "Deactivation" } & \multirow{2}{*}{\multicolumn{2}{|c|}{ No PGSS > PGSS }} & \multirow{2}{*}{\multicolumn{2}{|c|}{$\underline{\text { PGSS }>\text { No PGSS }}$}} \\
\hline & & \multicolumn{2}{|c|}{ No PGSS } & \multicolumn{2}{|c|}{ PGSS } & \multicolumn{2}{|c|}{ No PGSS } & \multicolumn{2}{|c|}{ PGSS } & & & & \\
\hline & & Left & Right & Left & Right & Left & Right & Left & Right & Left & Right & Left & Right \\
\hline \multirow[t]{10}{*}{ Frontal } & SupFrontDorsLat & & & & & & & 38 & 56 & & 24 & & \\
\hline & MidFrontLat & & & & & & & & 84 & & & & \\
\hline & InfFrontOper & 63 & 100 & 19 & & & & & 22 & 33 & 29 & & \\
\hline & InfFrontTri & 32 & 153 & & & & & & & 82 & 12 & & \\
\hline & SupFrontMed & & & & & & & 13 & 47 & 37 & 18 & & \\
\hline & SupFrontMedOrb & & & & & & & & 30 & & 15 & & \\
\hline & MidOrbFront & & & & & & 33 & & 68 & & & & \\
\hline & InfOrbFront & & & & & & 18 & & 88 & 57 & 104 & & \\
\hline & RolOper & & & & & & 6 & 74 & 90 & 3 & & & \\
\hline & Precentral & 160 & 201 & & & & & & & 170 & & & \\
\hline \multirow[t]{6}{*}{ Temporal } & SupTemporal & & 100 & & & & 61 & 195 & 207 & & & & \\
\hline & MidTemporal & 786 & 881 & 403 & 588 & & 27 & 227 & 225 & 165 & 212 & & \\
\hline & SupTempPole & & & 21 & & & & & 29 & 10 & & & \\
\hline & MidTempPole & & & & & & & & 16 & & & & \\
\hline & InfTemporal & 180 & 608 & 48 & 525 & & & 59 & 12 & 189 & & & \\
\hline & Heschl & & & & & & 13 & 11 & 17 & & & & \\
\hline \multirow[t]{6}{*}{ Parietal } & Postcentral & 19 & & & & & & & & 20 & 22 & & \\
\hline & SupParietal & 221 & 46 & 31 & & & & & & & & & \\
\hline & InfParietal & 301 & & 100 & & & 159 & 19 & 37 & & & & \\
\hline & Angular & 6 & 50 & & & & 255 & 258 & 387 & 6 & 17 & & \\
\hline & Supramarg & 89 & 12 & & & & 14 & 11 & 8 & 6 & & & \\
\hline & Precuneus & & 55 & & & & 18 & 18 & 75 & & & & \\
\hline \multirow[t]{7}{*}{ Occipital } & SupOccipital & 646 & 794 & 6 & 142 & & & & & 130 & 66 & & \\
\hline & MidOccipital & 1877 & 964 & 584 & 305 & & & 55 & & 689 & 15 & & \\
\hline & InfOccipital & 608 & 552 & 332 & 464 & & & & & 90 & 19 & & \\
\hline & Cuneus & 64 & 375 & & 134 & & & & & & 32 & & \\
\hline & Calcarine & 404 & 543 & 101 & 248 & & & 66 & 37 & 45 & 37 & & \\
\hline & Lingual & 272 & 491 & 34 & 37 & 93 & 25 & 101 & 105 & 28 & 189 & & \\
\hline & Fusiform & 557 & 624 & 344 & 347 & 217 & 159 & 372 & 373 & 445 & 141 & & \\
\hline \multirow[t]{2}{*}{ Cing } & AntCingulum & & & & & & & & 148 & & 47 & & \\
\hline & MidCingulum & & & & & & & & 28 & & & & \\
\hline \multirow[t]{6}{*}{ Subcortical } & HippFormation & 14 & 61 & & & 39 & 21 & 110 & 130 & 42 & 57 & & \\
\hline & Insula & 32 & & 14 & & & & & 87 & & & & \\
\hline & Amygdala & 9 & 5 & & & & & & & 4 & 8 & & \\
\hline & Thalamus & 7 & 82 & 11 & & & & 48 & 77 & & 35 & & \\
\hline & Basalganglia & 17 & 30 & & & & & & & 15 & 10 & & \\
\hline & Cerebellum & 166 & 394 & & 174 & 33 & & 213 & 38 & 84 & & & \\
\hline
\end{tabular}

Abbreviations: Cing, cingulum; Dors, dorsal; Front, frontal; Inf, inferior; Lat, lateral; Mid, middle; Oper, operculum; Orb, orbital; Rol, rolandic; Sup, superior; Supramarg, supramarginal; Temp, temporal; Tri, pars triangularis.

Cluster sizes of "PGSS vs. No PGSS" comparison for this contrast (as shown in Fig. 2) are given on the right. Brain regions are labeled according to automated anatomical labeling system (AAL; Tzourio-Mazoyer et al., 2002). Because the focus of the paper is on the comparison of the two streams of analyses with and without PGSS analysis, region of interest corrections were chosen to determine significance. One might be concerned about the region of interest criterion regarding the large number of structures listed in this table; however, most of these structures have been chosen as region of interest in previously published studies. Volumes are given as the overall number of voxel within all significant clusters in each region of interest (small volume of interest analysis with $P<$ 0.001 on voxel and $P<0.05$ on cluster level).

focusing on structures with moderate to small effect sizes. As shown in Table 1, examples of affected structures included not only visual processing structures in the parieto-temporo-occipital cortex, but also included to limbic and paralimbic structures.

With regard to signal decreases (see Fig. 1A and Table 1), the use of global signal scaling augmented effects in structures with a negative correlation of emotional arousal and BOLD signal (indicated by blue-hued colors). Specifically, areas revealing significant deactivation in the analysis without PGSS, such as parietal, occipital, and temporal structures, revealed enhanced effect sizes and cluster volumes in the PGSS analysis. In fact, the PGSS analysis revealed significant deactivations not observed when omitting global signal covariates, especially left parietal and right hemispheric subcortical structures. 
Low arousing emotional vs. neutral picture contrasts

Global signal did not significantly covary with low arousal and neutral picture conditions $[F(1,42)=0 ; n s]$. Consequently, as shown in Fig. 1B, the linear trend contrast for significant activations and deactivations as a function of low arousal emotional, compared to neutral, picture processing revealed similar effects for both streams of functional image analysis with and without PGSS. Slight differences in the outcome of both analyses were considered beyond the scope of the present report.

\section{No-PGSS vs. PGSS contrasts}

Second-level two-sample $t$ tests comparing the contrast analysis results of all subjects with and without PGSS served to more directly assess the effects of global scaling. Focusing on the "High Arousing Emotional vs. Neutral" picture contrast, significant differences between the functional analyses with and without PGSS were observed, including structures selectively activated by highly emotional compared to neutral picture processing (see top of Fig. 2 and Table 1). Furthermore, significant "deactivations" were observed for the analyses with PGSS relative to unscaled. Interestingly, there were no cases of opposing effects, i.e., stronger "activations" with application of PGSS, or stronger "deactivations" without global scaling correction.

Second-level analysis of the contrast comparing low arousal emotional and neutral picture processing confirmed that differences between analyses with and without PGSS were marginal when underlying assumptions were not violated (see bottom of Fig. 2).

\section{Effects of global scaling on gender effects and hemispheric} asymmetries

In addition to the pronounced correlation of global signal and experimental stimuli observed for the contrast of highly arousing emotional compared to neutral stimuli, the correlation was examined among different subgroups. Specifically, although significant for both genders, the correlation of global signal and the contrast "High Arousing Pleasant vs. Neutral" pictures appeared more pronounced in males $[F(1,10)=16.4 ; P<0.005]$ than in females $[F(1,11)=10.8 ; P<0.01]$. Under these circumstances, the

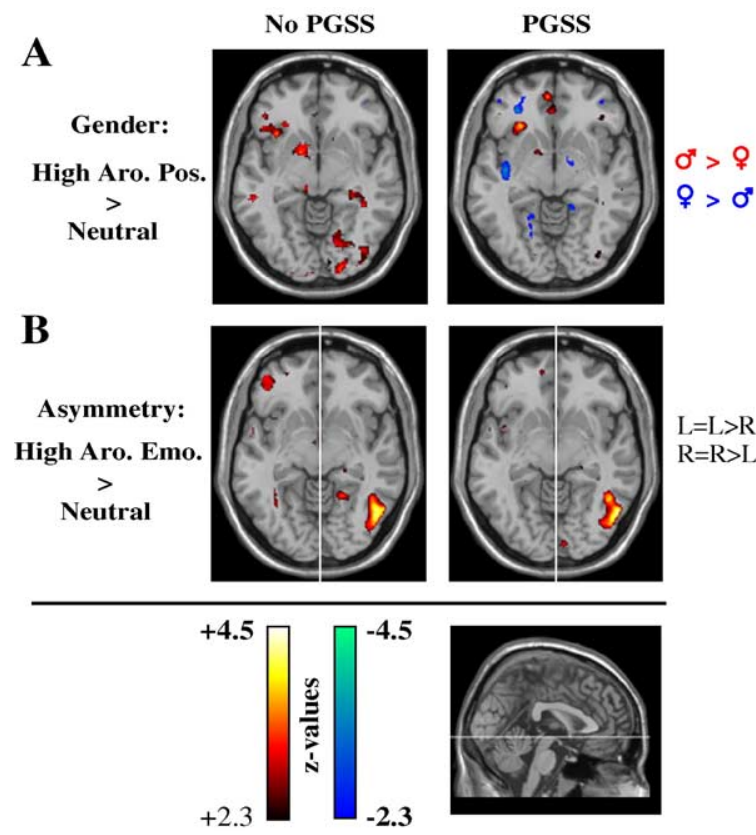

Fig. 3. (A) Second-level two-sample $t$ tests $(k=20$; DF $=20)$ of gender differences comparing males (10) with females (11) for the contrast "High Arousing Pleasant vs. Neutral” pictures. Red-hued colors represent significant stronger BOLD difference activations in males compared to females; blue hued colors indicate significantly stronger activations in females compared to males. (B) Second-level paired $t$ tests $(k=20$; DF $=$ $20)$ of hemispheric asymmetries contrasting the high arousing emotional with neutral picture condition (as illustrated in Fig. 1A) with their corresponding laterally transposed image. Activations on the left/right side represent a significant left/right hemispheric dominance.

application of PGSS might diminish gender differences, because PGSS effects are proportionally greater for males than females.

Replicating previous findings (see Fig. 3A), without application of PGSS, males showed stronger activations to high arousing emotional pictures than females in several cortical structures (e.g., the right extended visual cortex and left inferior frontal gyrus) as well as subcortical areas (e.g., right hippocampal formation, left basal ganglia). However, application of PGSS attenuated these gender differences, and in addition, amplified the opposite effect of

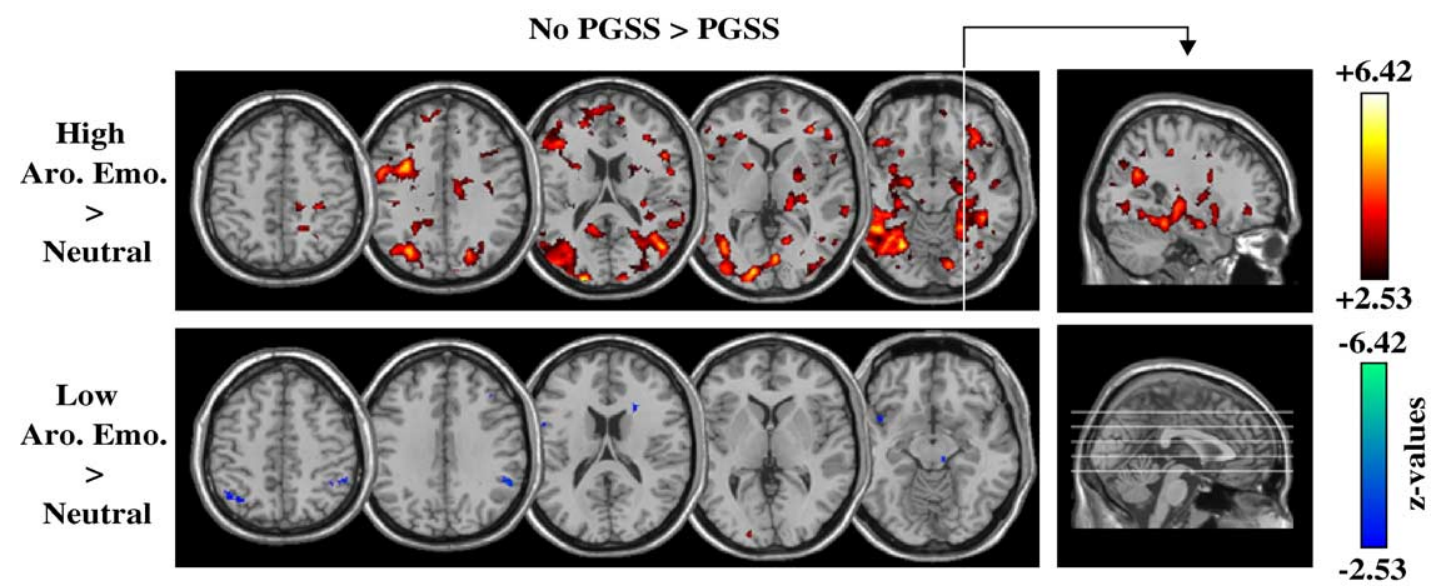

Fig. 2. Random effect analysis across 21 subjects $(k=20 ; \mathrm{DF}=20)$. Significant BOLD signal differences of the second level paired $t$ test comparing the contrasts as given in Fig. 1 with and without PGSS. 
greater activation to emotional pictures by females (e.g., in the left insula region).

The unjustified application of PGSS may also affect the observation of hemispheric asymmetries in emotion processing. Therefore, as shown in Fig. 3B, hemispheric asymmetries in the processing of highly arousing emotionally and neutral pictures were assessed with and without application of PGSS. The analysis without global scaling revealed a pronounced right-lateralized effect of emotional activation in inferior, mid-, and superior temporal regions, as well as a pronounced left-lateralized effect in the mid-orbitofrontal regions. In contrast, the PGSS-based analysis failed to reveal asymmetries in emotional activations in the orbitofrontal regions.

\section{Discussion}

The present report tested the underlying validity of using PGSS with a particular emphasis on tasks involving affective picture perception. The results revealed a strong positive relationship between global signal and the hemodynamic effects of processing highly arousing emotional pictures. Consequently, inferences about brain function were misleading regarding the processing of highly arousing emotional pictures after controlling for global hemodynamic changes using PGSS procedures. In contrast, when comparing low arousal and neutral picture conditions, no significant correlation between global signal and experimental conditions was observed. However, more research is needed to determine whether these findings generalize to other low arousal stimulus materials, i.e., facial expressions, and other experimental contexts.

Consistent with results of previous studies investigating cognitive tasks (cf. Aguirre et al., 1998; Desjardins et al., 2001), violations of the orthogonality assumption between global signal and experimental condition were reflected in quantitative and qualitative differences when comparing the outcome of the statistical analysis with and without application of PGSS. Focusing on sensory stimulus processing (cf. Bradley et al., 2003; Davis and Whalen, 2001; Pessoa et al., 2002), highly arousing emotional stimuli were associated with enhanced activation in the extended visual cortex in both scaled and unscaled analyses. However, confounds introduced by the unjustified use of global signal covariates greatly attenuated the extent of activation, as revealed by reduced cluster size and insensitivity to the recruitment of neural structures in the ventral and dorsal visual processing areas. With regard to the processing of emotionally arousing material in the visual processing streams, the PGSS analysis would therefore erroneously suggest localized and discrete rather than widely distributed neural activation.

Emotional stimuli have also been associated with activations in limbic and paralimbic structures (e.g., Hamann and Mao, 2002; Hamann et al., 2004; Morris et al., 1998; Pessoa et al., 2002; Vuilleumier et al., 2001). However, results were rather inconsistent regarding the emotion-driven activation of these structures across tasks (cf., e.g., Davis and Whalen, 2001; Phan et al., 2002). The present data suggest that the unjustified use of global signal scaling might contribute to these inconsistencies in results. In contrast to analyses omitting global scaling, the PGSS-based analysis used here failed to reveal significant activations in limbic and paralimbic regions during highly arousing picture perception. It seems noteworthy that the effect of global scaling was apparent in the amygdala, a key structure in emotional processing in current theories of emotional response (e.g., Lang et al., 2000; LeDoux, 2000). Thus, the unjustified application of global signal covariates has the potential to be a confound with regard to limbic and paralimbic activation, possibly contributing to inconsistencies in the literature.

It appears likely that the untested use of global signal covariates impedes generalization across studies, subject groups, and stimulus materials (Davis and Whalen, 2001; Phan et al., 2002; Wager et al., 2003). Particularly troublesome errors of interpretation might arise when comparing results of experimental conditions with and without significant global signal correlation. Pronounced distortions in the pattern of findings are introduced because the detrimental effects of the unjustified use of global signal covariates impinge on conditions with a strong correlation of global signal and task manipulation. The present study provided an example of this issue regarding the effects of emotional picture viewing (see Fig. 1). Similar falsifying effects might be expected when considering studies comparing subject groups which may differ in terms of effect sizes and/or signal to noise ratios within widely distributed neural regions. For instance, examining gender differences in emotion processing in the extended visual cortex, Sabatinelli et al. (2004) reported more pronounced extrastriate activation in males compared to females specifically when viewing highly arousing erotic pictures (an event-related fMRI study without global scaling). The present data replicated these gender differences for the extended right visual cortex (Fig. 3A), however, only in the analysis without PGSS. The unjustified application of PGSS diminished gender differences in the extended visual cortex (as well as limbic and prefrontal regions) because the correlation of global signal and experimental condition was more pronounced for males than females. For similar reasons, the use of PGSS also distorted the pattern of hemispheric activation associated with emotional picture processing. Specifically, the PGSS-based analysis failed to reveal asymmetries in emotional activations in midorbitofrontal regions (Fig. 3B). Thus, the unjustified use of PGSS might contribute to the mixed findings regarding lateralization effects in emotional stimulus processing (Wager et al., 2003).

Taken together, the present study demonstrated that the concerns and precautions regarding the standard use of PGSS in functional imaging of the cognitive domain are particularly relevant to emotion research. The unjustified application of PGSS in emotion research has the potential to distort statistical findings in key structures implicated in emotional processing, and may contribute to conflicting results in affective fMRI studies, particularly with respect to limbic and paralimbic structures. Reiterating Aguirre et al. (1998), it is recommended that authors report the correlation of global signal and experimental condition when using PGSS, and omit this confound in cases in which the global signal and experimental condition show a significant relationship.

\section{Acknowledgments}

This research was supported by the Deutsche Forschungsgemeinschaft (For348-J; Schu1074/10-3) and the Academy of Science, Heidelberg (WIN-program).

\section{References}

Aguirre, G.K., Zarahn, E., D'Esposito, M., 1998. The inferential impact of global signal covariates in functional neuroimaging analyses. NeuroImage 8, 302-306. 
Andersson, J.L., 1997. How to estimate global activity independent of changes in local activity. NeuroImage 6, 237-244.

Arndt, S., Cizadlo, T., O'Leary, D., Gold, S., Andreasen, N.C., 1996. Normalizing counts and cerebral blood flow intensity in functional imaging studies of the human brain. NeuroImage 3, 175-184.

Bradley, M.M., Sabatinelli, D., Lang, P.J., Fitzsimmons, J.R., King, W., Desai, P., 2003. Activation of the visual cortex in motivated attention. Behav. Neurosci. 117, 369-380.

Davis, M., Whalen, P.J., 2001. The amygdala: vigilance and emotion. Mol. Psychiatry 6, 13-34.

Desjardins, A.E., Kiehl, K.A., Liddle, P.F., 2001. Removal of confounding effects of global signal in functional MRI analyses. Neurolmage 13, $751-758$

Fox, P.T., Mintun, M.A., Reiman, E.M., Raichle, M.E., 1988. Enhanced detection of focal brain responses using intersubjects and change distribution analysis of subtracted PET images. J. Cereb. Blood Flow Metab. 8, 642-653.

Friston, K.J., Frith, C.D., Liddle, P.F., Dolan, R.J., Lammertsma, A.A., Frackowiak, R.S., 1990. The relationship between global and local changes in PET scans. J. Cereb. Blood Flow Metab. 10, 458-466.

Friston, K.J., Holmes, A.P., Worsley, K.J., Poline, J.P., Frith, C.D., Frackowiak, R.S., 1995. Statistical parametric maps in functional imaging: a general linear approach. Hum. Brain Mapp. 2, 189-210.

Hamann, S., Mao, H., 2002. Positive and negative emotional verbal stimuli elicit activity in the left amygdala. NeuroReport 13, 15-19.

Hamann, S., Herman, R.A., Nolan, C.L., Wallen, K., 2004. Men and women differ in amygdala response to visual sexual stimuli. Nat. Neurosci. 7, $411-416$.

Lang, P.J., Bradley, M.M., Fitzsimmons, J.R., Cuthbert, B.N., Scott, J.D., Moulder, B., Nangia, V., 1998. Emotional arousal and activation of the visual cortex: an fMRI analysis. Psychophysiology 35, 199-210.

Lang, P.J., Bradley, M.M., Cuthbert, B.N., 1999. International Affective Picture System (IAPS): Technical Manual and Affective Ratings. The Center for Research in Psychophysiology, Gainesville, FL.

Lang, P.J., Davis, M., Ohman, A., 2000. Fear and anxiety: animal models and human cognitive psychophysiology. J. Affect Disord. 61, $137-159$

LeDoux, J.E., 2000. Emotion circuits in the brain. Annu. Rev. Neurosci. 23, $155-184$.
Morris, J.S., Ohman, A., Dolan, R.J., 1998. Conscious and unconscious emotional learning in the human amygdala. Nature 393, 467-470.

Pessoa, L., Kastner, S., Ungerleider, L.G., 2002. Attentional control of the processing of neural and emotional stimuli. Brain Res. Cogn. Brain Res. $15,31-45$.

Phan, K.L., Wager, T., Taylor, S.F., Liberzon, I., 2002. Functional neuroanatomy of emotion: a meta-analysis of emotion activation studies in PET and fMRI. NeuroImage 16, 331-348.

Ramsay, S.C., Murphy, K., Shea, S.A., Friston, K.J., Lammertsma, A.A., Clark, J.C., Adams, L., Guz, A., Frackowiak, R.S., 1993. Changes in global cerebral blood flow in humans: effect on regional cerebral blood flow during a neural activation task. J. Physiol. 471, 521-534.

Rosenthal, R., Rosnow, R., 1985. Contrast Analysis: Focused Comparisons in the Analysis of Variance. CUP, Cambridge.

Sabatinelli, D., Flaisch, T., Bradley, M.M., Fitzsimmons, J.R., Lang, P.J., 2004. Affective picture perception: gender differences in visual cortex? NeuroReport 19, 1109-1112.

Scheffé, H., 1959. The analysis of variance. Wiley, New York.

Strother, S.C., Anderson, J.R., Schaper, K.A., Sidtis, J.J., Liow, J.-S., Woods, R.P., Rottenberg, D.A., 1995. Principal component analysis and the scaled subprofile model compared to intersubject averaging and statistical parametric mapping: I. "Functional connectivity" of the human motor system studied with [15O] water PET. J. Cereb. Blood Flow Metab. 15, 738-753.

Tzourio-Mazoyer, N., Landeau, B., Papathanassiou, D., Crivello, F., Etard, O., Delcroix, N., Mazoyer, B., Joliot, M., 2002. Automated anatomical labeling of activations in SPM using a macroscopic anatomical parcellation of the MNI MRI single-subject brain. NeuroImage 15, $273-289$.

Vuilleumier, P., Armony, J.L., Driver, J., Dolan, R.J., 2001. Effects of attention and emotion on face processing in the human brain: an eventrelated fMRI study. Neuron 30, 829-841.

Wager, T.D., Phan, K.L., Liberzon, I., Taylor, S.F., 2003. Valence, gender, and lateralization of functional brain anatomy in emotion: a metaanalysis of findings from neuroimaging. NeuroImage 19, 513-531.

Whalen, P.J., Shin, L.M., McInerney, S.C., Fischer, H., Wright, C.I., Rauch, S.L., 2001. A functional MRI study of human amygdala responses to facial expressions of fear versus anger. Emotion 1, $70-83$. 